\title{
Initial CT blend sign is not associated with poor outcome in patients following stereotactic minimally invasive surgery
}

\author{
Xu Yang \\ Guiyang First People`s Hospital \\ Yan Zhu \\ Shanghai Second Rehabilitation Hospital \\ Linshan Zhang \\ Affiliated Hospital of Guizhou Medical University \\ Likun Wang \\ Affiliated Hospital of Guizhou Medical University \\ Yuanhong Mao \\ Affiliated Hospital of Guizhou Medical University \\ Yinghui $\mathrm{Li}$ \\ Affiliated Hospital of Guizhou Medical University \\ Jingbiao Luo \\ Guangzhou First People`s Hospital \\ Guofeng Wu ( $\nabla$ wuguofeng3013@sina.com) \\ Guizhou Medical University
}

Research article

Keywords: Intracerebral haemorrhage, Stereotactic techniques, Minimally invasive surgery, CT Blend sign, Glassgow Coma Scale

Posted Date: January 19th, 2021

DOI: https://doi.org/10.21203/rs.3.rs-37415/v3

License: @) (7) This work is licensed under a Creative Commons Attribution 4.0 International License. Read Full License

Version of Record: A version of this preprint was published at BMC Neurology on April 15th, 2021. See the published version at https://doi.org/10.1186/s12883-021-02181-0. 


\section{Abstract}

Background: The initial computed tomography (CT) blend sign has been used as an imaging marker to predict haematoma expansion and poor outcomes in patients with a small volume intracerebral haemorrhage (ICH). However, the relationship between the blend sign and outcomes remains elusive. The present study aimed to retrospectively measure the impact of initial CT blend signs on short-term outcomes in patients with hypertensive ICH who underwent stereotactic minimally invasive surgery (sMIS).

Methods: We enrolled 242 patients with spontaneous ICH. Based on the initial CT features, the patients were assigned to a blend sign group (91 patients) or a nonblend sign (control) group (151 patients). The NIHSS, GCS and mRS were used to measure the effects of sMIS. The rates of severe pulmonary infection and cardiac complications were also compared between the two groups.

Results: No significant differences in NIHSS and GCS scores were observed between the two groups. The proportion of patients with good outcomes during follow-up was not different between the two groups. The rate of rehaemorrhaging increased in the blend sign group. No significant differences in severe pulmonary infections and cardiac complications were noted between the two groups.

Conclusions: The initial CT blend sign was not associated with poor outcomes in patients with hypertensive ICH who underwent sMIS. ICH patients with CT blend signs should undergo sMIS if they are suitable candidates for surgery.

\section{Background}

Spontaneous ICH is a devastating life-threatening disease with high global mortality and morbidity worldwide. To improve the outcomes of patients with ICH, various clinical medical and surgical trials for interventions for $\mathrm{ICH}$ have been conducted in the past 10 years ${ }^{1}$. However, although research and trials of therapies for ICH have increased greatly, the 30 -day mortality remains as high as $40 \%$ worldwide ${ }^{2}$. No interventional therapy has been demonstrated to be effective in improving outcomes ${ }^{3}$. Open craniotomy haematoma evacuation in large clinical randomized trials has not shown benefits for patients with ICH ${ }^{4}$. Although craniotomy showed effectiveness in removing $\mathrm{ICH}$, it resulted in substantial brain injury complicated by pulmonary infection ${ }^{4}$. The advantages of conventional surgical management over conservative medications for hypertensive $\mathrm{ICH}$ are controversial ${ }^{5}$. Patients with supratentorial ICH showed no overall benefit from early neurosurgical management compared with initial conservative treatment ${ }^{6}$. Brain injury due to conventional surgical procedures for ICH might counteract the potential benefits of haematoma removal during open surgery ${ }^{7}$. Recently, MIS for ICH management has been evaluated in numerous clinical trials and has achieved favourable results ${ }^{8-10}$. Minimally invasive puncture and drainage are the least traumatic procedures and have the shortest operative times ${ }^{4}$. However, for moderate to large $\mathrm{ICH}$, minimally invasive catheter evacuation followed by thrombolysis did not improve the proportion of patients who achieved a good response, and a haematoma size reduction to $15 \mathrm{ml}$ or less was associated with improved mRS scores at 365 days in patients who were stabilized ${ }^{4}$.

Haematoma expansion (HE) or haematoma growth predicts substantially worse prognosis and might be potentially preventable if high-risk patients could be identified in the early stage of $\mathrm{ICH}^{2}$. Imaging markers, such as the blend signs, black hole signs and spot signs, have been identified as predicting $\mathrm{HE}^{2}$, 8-10. The blend sign showed an association with poor outcome in patients with a small volume of ICH treated with medications ${ }^{2}$. Our previous studies showed that the black hole sign and the blend sign predicted rehaemorrhage in patients with hypertensive ICH who underwent stereotactic minimally invasive surgery (sMIS) 11,12. However, whether the initial CT blend signs are associated with poor outcome in patients following sMIS remains unknown. We speculated that the initial CT blend signs are associated with poor outcome in patients with $\mathrm{ICH}$ receiving sMIS. The present study aimed to retrospectively observe the influence of the initial CT blend sign on outcomes in patients with spontaneous ICH following sMIS.

\section{Methods}

The Ethics Committee of the Affiliated Hospital of Guizhou Medical University approved this retrospective study. The study was performed based on the WMA Declaration of Helsinki. Patients with ICH admitted to our hospital who underwent sMIS were included in our study. The recruitment period was from January 1,2018 , to June $30,2019$.

\section{Study design and participants}

\section{Study design}

A retrospective analysis was performed. The authors aimed to determine whether initial CT blend signs were associated with poor functional outcome of patients with ICH following sMIS. We collected data from patients with ICH by reviewing the medical records of the Affiliated Hospital of Guizhou Medical University. The recruitment period was from January 1, 2018, to June 30, 2019. The patients were diagnosed using a baseline CT scan within 1 hour of admission, and surgery was performed within 27 hours of admission. The eligible patients with ICH were selected according to the inclusion criteria listed below. All eligible patients were treated by sMIS and were assigned to two groups based on their haematoma features.

The inclusion criteria were as follows: (1) patients over 18 years old with a history of hypertension or hypertension observed upon admission as well as symptoms and signs meeting the diagnostic criteria for ICH, which was confirmed using a nonenhanced CT scan; (2) patients who suffered from spontaneous $\mathrm{ICH}$ in the supratentorial area (the basal ganglia, thalamus or cerebral lobes); (3) patients with ICH volumes between $30 \mathrm{ml}$ and $50 \mathrm{ml}$; (4) patients with no contraindications for surgery; and (4) the authorized representatives of the patients provided consent for surgery. 
The exclusion criteria were the same as those in previously published studies ${ }^{12}$. Patients with ICH located in the brainstem or with secondary ICH from haemorrhagic transformation from brain infarction were not included. Patients without authorized representative consent to surgery were also excluded from the study.

\section{Participants}

From January 1, 2018, to June 30, 2019, a total of 710 patients with spontaneous ICH were admitted to the Affiliated Hospital of Guizhou Medical University. Among them, 318 patients underwent sMIS. Of the 318 patients who underwent sMIS, 25 left the hospital within one week without medical orders, 21 patients experienced ICH in the brainstem, and another 30 patients displayed large-volume (over $50 \mathrm{~mL}$ ) ICH on CT. These 76 patients were not included in the final analysis (Fig. 1).

Based on the inclusion criteria, 242 consecutive patients with spontaneous ICH were included in the present study. All patients in the present study underwent sMIS. The patients were assigned to the following groups based on their CT haematoma features: the blend sign group included 91 patients, and the nonblend sign group (control group) included 151 patients with spontaneous ICH. The baseline clinical characteristics of the patients are listed in Table 1.

\section{Imaging analysis}

The initial and follow-up CT scans (General Electric Medical Systems, Milwaukee, WI) were performed using standard clinical parameters with axial 3-mmthick sections, a current of $225 \mathrm{~mA}$, a window level of 39 and a window width of 120 . The images were obtained and stored for further evaluation. The ICH for each patient was located in the supratentorial area (including the basal ganglia, thalamus or cerebral lobes). Two experts (one neurosurgical expert and one neuroimaging expert) who were blinded to the clinical information served as reviewers and independently evaluated the shape features of the haematomas. The shape of the haematoma was assessed by visual inspection ${ }^{16}$. The blend sign was determined by the criteria proposed in previously published studies ${ }^{13}$. Briefly, the haematoma blend sign was defined as follows: (1) blending of a relatively hypoattenuating area with an adjacent hyperattenuating region within a haematoma; (2) presence of a well-defined margin between the hypoattenuating area and adjacent hyperattenuating region that is easily recognized by the naked eye; (3) the haematoma should have at least an 18 Hounsfield unit difference between the 2 density regions; and (4) the relatively hypoattenuating area was not encapsulated by the hyperattenuating region.

Discrepancies about the presence of the blend signs were settled by joint discussion between the readers.

Haematoma volumes were estimated based on $\mathrm{CT}$ using the $\mathrm{ABC} / 2$ formula $(t=\pi / 6 \times 1 \times s \times \text { slice })^{14}$. The criteria for identifying the blend sign were the same as those reported in the literature ${ }^{13}$. The blend sign was composed of two parts with different densities on CT (Fig. 2).

\section{Patient treatment}

\section{sMIS for ICH evacuation}

The sMIS procedures for ICH evacuation were the same as those used in our previously published studies ${ }^{19,22,23}$. To remove the influences of surgical technical factors on the outcomes, surgical procedures were performed by two experienced neurosurgeons. Briefly, a stereotactic instrument was fixed on the patient's skull, and a repeated CT scan was performed for each patient prior to surgery. After the repeating CT scan was performed, the patient was transferred to the operating room. Using the CT scan, the coordinates of the ICH were determined, and we punctured the skull using a 3-mm-diameter needle (with a drill integrated into the needle guard) under the guidance of the stereotactic instrument. After the drill was replaced by a blunt-tip plastic needle core, the LY-1-type puncture-needle set was inserted slightly into the haematoma. Following removal of the plastic-needle core, the liquid part of the haematoma was aspirated using a 10-ml syringe (Fig. 3). The aspiration was stopped after the first resistance was encountered, and the needle guard connected to a plastic tube was retained for several days for drainage. The patients were transferred to the intensive care unit after removing the location framework and stereotactic apparatus. Then, 50,000 units of urokinase (diluted in $2 \mathrm{ml}$ of normal saline) were injected slowly every 8 hours into the residual haematoma area to dissolve the solid part of the haematoma. The needle system was closed for 2 hours before reopening to allow spontaneous drainage. The first postoperative follow-up CT scan was performed on the day following surgery, and the second postoperative CT was performed on the third day after surgery. Some patients needed a third or even a fourth postoperative follow-up CT scan. If the patients showed neurological deterioration at any time after surgery, a repeated CT scan was performed.

\section{Medications}

All patients in our study received the same medical management based on the guidelines for the treatment of hypertensive ICH ${ }^{15}$. More comprehensive measures were also taken in all patients, including the prevention of deep-venous thrombosis (DVT), the control of temperature and blood glucose, nutritional support, and the prevention of other complications. The main measures used for preventing DVT were to move the paralysed limbs slowly and to wear socks. No anticoagulants were used to prevent DVT during the hospital stay because they might induce haemorrhage.

\section{Functional outcomes}

The primary functional outcome was a good functional outcome, defined as the proportion of patients who achieved a modified Rankin Scale (mRS) score of 0-3 at discharge. The mRS was conducted by neurological experts blinded to both the study and the imaging. The secondary outcomes included the National Institutes of Health Stroke Scale (NIHSS) scores, the Glasgow Coma Scale (GCS) scores and the ICH volume changes. The outcome was considered

favourable if the mRS score was 0-3 points. In contrast, if the mRS score was $>3$ points, the outcome was considered poor ${ }^{4}$. The GCS and NIHSS scores were assessed upon admission and at one and two weeks after surgery by experienced neurological experts. Mortality and complications were recorded during the hospital stay and were compared between the two groups. 


\section{Complications}

Some patients suffered from life-threatening complications during their hospital stays. Severe cardiopulmonary complications included severe pulmonary infection, respiratory failure, and heart failure. The cardiopulmonary complications were those that occurred during their hospital stay. Exacerbations of chronic heart failure and respiratory failure, as well as community-acquired pneumonia, were not included.

Postoperative rehaemorrhaging was defined as when the ICH (hyperdensity) reappeared in the haematoma region on the follow-up $\mathrm{CT}$ scan after it was removed completely following surgery ${ }^{12}$. An increase in the haematoma volume of $>33 \%{ }^{16}$ compared with the $\mathrm{ICH}$ volume determined by using the previous CT scan, which showed significantly decreased ICH volume after sMIS, was also considered a case of postoperative rehaemorrhage.

\section{Statistical analysis}

On the basis of the assumption that $25 \%$ of patients would have a mRS score of $0-3$ in the blend sign group versus $45 \%$ of patients would have a mRS score of 0-3 following sMIS in the control group ${ }^{4}$, we estimated that 90 patients in each group would provide $81.0 \%$ statistical power at an a level of 0.05 . The permissible error $d$ was 0.1 .

A commercially available software package (SPSS, Version 22.0) was used to perform the statistical analyses. Categorical data are expressed as proportions, and continuous variables are presented as the mean and SD. Demographic, clinical, and radiological characteristics were compared between patients with shape-regular or shape-irregular ICH using Student's $t$ tests (for normal distribution) or nonparametric tests (if the data were not normally distributed). A difference in the GCS and NIHSS scores between different time points was analysed using the method of repeated measures. A p value less than 0.05 was considered to indicate a statistically significant difference. The independent association between the initial CT blend sign and the outcome of patients after sMIS was evaluated using binary logistic regression. The interobserver reliability of the CT blend sign was assessed by calculating the $\mathrm{k}$ values. The $\mathrm{k}$ values were categorized as reported in the literature ${ }^{12}$. A $\mathrm{k}$ value equal to 1 indicated total agreement between the observers.

\section{Results}

\section{The baseline data}

During the recruitment period, 318 patients were assessed for eligibility. Of the 318 patients, 242 with ICH met our inclusion criteria. One hundred eighty patients were men, and 69 were women. The ages ranged from 31 to 93 years, with an average of $57.05 \pm 12.703$. The time from onset to baseline CT was 5.0 (2.0-9.7) hours. The mean admission GCS score was 11 (8-13), and the mean NIHSS score was 16 (14-20). One hundred eighty-four patients showed haematoma in the basal ganglia area, 34 patients in the cerebral lobes, and 24 patients in the thalamus.

Based on their haematoma features, the 242 included patients with ICH were assigned to the abovementioned two study groups. No significant differences were noted between the blend sign group and the control group in age, history of smoking, drinking, preoperative ICH volume, anticoagulants, GCS score on admission, NIHSS score on admission, time from onset to admission, time from onset to baseline $\mathrm{CT}$, and time from onset to surgery. Only the blend sign group showed a higher rate of hypertension history (Table 1).

Discrepancies between the neurosurgeon and the radiologist were noted in 3 patients. The interobserver agreement for identifying the shape features of the haematoma was good and reliable between the 2 readers, with a $\mathrm{k}$ value of 0.974 and a $95 \%$ confidence interval of 0.94-1.00.

\section{Changes in haematoma volume}

Compared with the control group, the blend sign group did not show significant changes in the ICH volume or the time for removal of the drainage tube. The rates of $\mathrm{ICH}$ clearance between the blend sign group and the nonblend sign group were also similar. No significant differences were observed between the two groups (Fig. 4, Table 2). These findings demonstrated that the blend sign did not affect the removal of ICH by sMIS.

\section{Changes in the GCS and the NIHSS}

The GCS and the NIHSS were determined at one and two weeks after surgery. The blend sign group and the control group showed significantly greater GCS and lower NIHSS at one and two weeks after surgery compared with those on admission (Tables 3 and 4). However, no significant difference was observed between the two groups. These findings suggested that patients with the blend sign on initial CT would obtain the same short-term outcome as nonblend sign patients after sMIS.

\section{Complications}

The blend sign group showed similar rates of severe complications, including pulmonary infection and heart failure, compared with the control group ( $P>0.05$, Table 5). However, the blend sign group showed a higher rate of rehaemorrhage than the control group $(P=0.049)$.

\section{Influences of the CT blend sign on the outcome following SMIS}

Of the 91 patients with CT blend signs, 50 (54.9\%) showed good outcomes. In 151 patients without blend signs, 71 (51.8\%) showed good outcomes. No significant differences between the two groups were observed. In 128 patients with good outcomes, 50 (39.1\%) had blend signs on the initial CT scan. To determine whether the CT blend signs were associated with poor outcomes, we performed a univariate analysis first and then conducted a binary logistic regression. The history of hypertension $(P=0.037)$, NIHSS score upon admission $(P<0.001)$, and GCS score upon admission $(P<0.001)$ showed statistical 
significance (Table 6). The blend sign showed no statistical significance with the poor outcome. Therefore, only the history of hypertension, the initial NIHSS score and the GCS score went into the binary logistic regression model. The final results suggested that the initial NIHSS score or the GCS score was an independent predictor of poor functional outcome in patients with ICH following sMIS (Table 7).

\section{Discussions}

Spontaneous ICH is the most common subtype of haemorrhagic stroke. The incidence of $\mathrm{ICH}$ accounts for approximately $10 \%-30 \%$ of all types of stroke worldwide. HE predicts substantially poor outcomes and is potentially preventable if high-risk patients could be identified in the early stage of ICH ${ }^{8}$. The initial $\mathrm{CT}$ blend sign could predict $\mathrm{HE}$ and was associated with poor outcome in patients who received medication management ${ }^{17}$. The blend signs also showed a close association with postoperative rebleeding in patients with $\mathrm{ICH}$ following $\mathrm{SMIS}^{12}$.

Minimally invasive procedures have been used to treat patients with $\mathrm{ICH}$ for more than ten years. These procedures were shown to remove ICH with minimal traumatic brain injury and to be beneficial for neurofunctional recovery ${ }^{18,19}$. Minimally invasive catheter aspiration of ICH followed by medications for dissolving the clot could be another choice of surgical approach as a therapeutic strategy for $\mathrm{ICH}^{20}$. Minimally invasive puncture and drainage showed the least trauma to the brain and had the shortest operative time ${ }^{4}$. Our previously published studies demonstrated that the initial CT blend signs showed a close association with postoperative rehaemorrhage in ICH patients following sMIS ${ }^{12}$. Therefore, we postulated that the blend signs could affect the outcome of patients with ICH following sMIS. In the present study, the GCS, NIHSS, mRS and postoperative complications were used as indexes to evaluate the outcome. However, the authors were unable to obtain the expected results. The GCS increased and the NIHSS decreased significantly at two weeks after surgery compared with those on admission. However, there were no significant differences between the blend sign group and the control group. The proportions of patients with favourable outcomes were compared between the patients with blend signs and the control subjects, and no significant difference was observed. Secondary complications after ICH are associated with prognosis ${ }^{21}, 22$. Pneumonia was the most common medical complication (15.1\%) after ICH ${ }^{23}$. Cardiac complications (5.9\%) also often occur after ICH due to neuroendocrine changes such as changes in catecholamine levels and elevated levels of brain natriuretic peptide

In the present study, the patients with blend signs following sMIS had similar rates of severe pulmonary infection and heart failure as those without blend signs. No significant difference was observed between the two groups, suggesting that the blend sign was not associated with the rate of complications following sMIS. The blend sign group showed a higher rate of postoperative rehaemorrhage than in our previously published study ${ }^{12}$. Although the blend signs predicted poor outcome in patients with small volumes of $\mathrm{ICH}$, no evidence demonstrates that blend signs were associated with poor outcome in patients following sMIS. sMIS should be performed to treat patients with blend signs on initial CT scans if the ICH volume is large enough and the patients are suitable candidates for surgery.

There were some limitations in the present study. The patients were not followed up after discharge. Therefore, we were unable to observe the long-term outcomes. Some patients were discharged from the hospital without medical orders, and mortality could not be recorded and compared, as no deaths occurred during their hospital stay. The present study was retrospective; randomized prospective studies with larger sample sizes are required in the future.

\section{Conclusions}

In conclusion, sMIS could remove intracerebral haematomas effectively. The initial CT blend signs are not associated with poor outcomes among patients with ICH following sMIS. ICH patients with CT blend signs obtained the same outcome as patients without the CT blend sign after sMIS.

\section{Abbreviations}

ICH: intracerebral haemorrhage; CT: computed tomography; sMIS: stereotactic minimally invasive surgery; GCS: Glasgow Coma Scale; NIHSS: National Institute of Health Stroke Scale

\section{Declarations}

\section{Acknowledgements}

We are grateful for the help provided by the Image Department of the Affiliated Hospital of Guizhou Medical University in the analysis of the computed tomography scans. We also wish to thank all the postgraduates who were involved in this study for their hard work.

\section{Funding}

This research was supported by the Natural Science Foundation of China (81971126/H0906), the Medical Speciality and Community Project Construction in Baoshan District - Neurorehabilitation Speciality (BSZK-2018-A01) as well as the High-level Overseas Talents Innovation and Entrepreneurship Merit-based Funding Projects $₫(2020) 05 \rrbracket$. The funding body did not take part in the design of the study or the collection, analysis, and interpretation of data or in the writing of the manuscript.

\section{Consent for publication}

Not applicable.

\section{Competing of interests}


The authors declare that they have no competing interests.

\section{Authors' contributions}

GW, LW and JL conceived of the study, participated in the design of the study, coordinated the study and drafted the manuscript. XY, LZ, YL and YM conducted the clinical study. YZ and LW performed the statistical analyses and revised the manuscript. All the authors read and approved the final manuscript.

\section{Availability of data and materials statement}

The datasets analysed in the current study are available from the corresponding author upon reasonable request.

\section{Ethics approval and consent to participate}

All the patients' authorized representatives and those patients who had the ability to communicate with the doctors agreed to participate in the study. Informed consent was obtained in written form.

\section{References}

1. Kim JY, Bae HJ. Spontaneous intracerebral hemorrhage: Management. J Stroke. 2017;19:28-39

2. Chen S, Zhao B, Wang W, Shi L, Reis C, Zhang J. Predictors of hematoma expansion predictors after intracerebral hemorrhage. Oncotarget. 2017;8:8934889363

3. Wilkinson DA, Pandey AS, Thompson BG, Keep RF, Hua Y, Xi G. Injury mechanisms in acute intracerebral hemorrhage. Neuropharmacology. 2018;134:240248

4. Hanley DF, Thompson RE, Rosenblum M, Yenokyan G, Lane K, McBee N, Mayo SW, Bistran-Hall AJ,et al. Efficacy and safety of minimally invasive surgery with thrombolysis in intracerebral haemorrhage evacuation (mistie iii): A randomised, controlled, open-label, blinded endpoint phase 3 trial. Lancet. 2019;393:1021-1032

5. Kim HT, Lee JM, Koh EJ, Choi HY. Surgery versus conservative treatment for spontaneous supratentorial intracerebral hemorrhage in spot sign positive patients. J Korean Neurosurg Soc.58:309-315

6. Mendelow AD, Gregson BA, Fernandes HM, Murray GD, Teasdale GM, Hope DT, Karimi A, et al. Early surgery versus initial conservative treatment in patients with spontaneous supratentorial intracerebral haematomas in the international surgical trial in intracerebral haemorrhage (stich): A randomised trial. Lancet. 2005;365:387-397

7. Labib MA, Shah M, Kassam AB, Young R, Zucker L, Maioriello A, Britz G, Agbi C, Day JD, etal. The safety and feasibility of image-guided brainpathmediated transsulcul hematoma evacuation: A multicenter study. Neurosurgery.80:515-524

8. Huang YW, Yang MF. Combining investigation of imaging markers (island sign and blend sign) and clinical factors in predicting hematoma expansion of intracerebral hemorrhage in the basal ganglia. World Neurosurg. 2018;120:e1000-e1010

9. Li Q, Yang WS, Wang XC, Cao D, Zhu D, Lv FJ, Liu Y, Yuan L, Zhang G, Xiong X. Blend sign predicts poor outcome in patients with intracerebral hemorrhage. Plos One. 2017;12:e0183082

10. Yu Z, Zheng J, Guo R, Ma L, Li M, Wang X, Lin S, Li H, You C. Performance of blend sign in predicting hematoma expansion in intracerebral hemorrhage: A meta-analysis. Clin Neurol Neurosurg. 2017;163:84-89

11. Shen Z, Wang L, Wu G, Li Q, Ren S, Mao Y. Computed tomographic black hole sign predicts postoperative rehemorrhage in patients with spontaneous intracranial hemorrhage following stereotactic minimally invasive surgery. World Neurosurg. 2018;120:e153-e160

12. Wu G, Shen Z, Wang L, Sun S, Luo J, Mao Y. Post-operative re-bleeding in patients with hypertensive ich is closely associated with the ct blend sign. $B M C$ Neurol. 2017;17:131

13. Li Q, Zhang G, Huang YJ, Dong MX, Lv FJ, Wei X, Chen JJ, Zhang LJ, Qin XY, Xie P. Blend sign on computed tomography: Novel and reliable predictor for early hematoma growth in patients with intracerebral hemorrhage. Stroke. 2015;46:2119-2123

14. Won SY, Zagorcic A, Dubinski D, Quick-Weller J, Herrmann E, Seifert V, Konczalla J. Excellent accuracy of abc/2 volume formula compared to computerassisted volumetric analysis of subdural hematomas. PLoS One.13:e0199809

15. Hemphill JC, 3rd, Greenberg SM, Anderson CS, Becker K, Bendok BR, Cushman M, Fung GL, Goldstein JN, Macdonald RL, Mitchell PH, Scott PA, Selim MH, Woo D. Guidelines for the management of spontaneous intracerebral hemorrhage: A guideline for healthcare professionals from the american heart association/american stroke association. Stroke. 2015;46:2032-2060

16. Brott T, Broderick J, Kothari R, Barsan W, Tomsick T, Sauerbeck L, Spilker J, Duldner J, Khoury J. Early hemorrhage growth in patients with intracerebral hemorrhage. Stroke. 1997;28:1-5

17. Li Q, Yang WS, Wang XC, Cao D, Zhu D, Lv FJ, Liu Y, Yuan L, Zhang G, Xiong X, Li R, Hu YX, Qin XY, Xie P. Blend sign predicts poor outcome in patients with intracerebral hemorrhage. PLoS One. 2017;12:e0183082

18. Li G, Qin X, Pen G, Wu W, Yang J, Yang Q. Effect of minimally invasive aspiration in treatment of massive intracerebral hemorrhage. Acta Neurochirurgica Supplement. 2011;111:381

19. Wartenberg KE, Mayer SA. Ultra-early hemostatic therapy for intracerebral hemorrhage: Future directions. Frontiers of Neurology \& Neuroscience. 2015;37:107 
20. Hanley DF, Thompson RE, Muschelli J, Rosenblum M, McBee N, Lane K, Bistran-Hall AJ, Mayo SW, Keyl P, et al. Safety and efficacy of minimally invasive surgery plus alteplase in intracerebral haemorrhage evacuation (mistie): A randomised, controlled, open-label, phase 2 trial. Lancet Neurol. 2016;15:12281237

21. Putaala J, Lehto M, Meretoja A, Silvennoinen K, Curtze S, Kääriäinen J, Koivunen RJ, et al. In-hospital cardiac complications after intracerebral hemorrhage. International Journal of Stroke Official Journal of the International Stroke Society. 2014;9:741-746

22. Shibazaki K, Kimura K, Sakai K, Aoki J, Sakamoto Y. Plasma brain natriuretic peptide is elevated in the acute phase of intracerebral hemorrhage. Journal of Clinical Neuroscience Official Journal of the Neurosurgical Society of Australasia. 2014;21:221-224

23. Stein M, Hamann GF, Misselwitz B, Uhl E, Kolodziej M, Reinges MH. In-hospital mortality and complication rates in surgically and conservatively treated patients with spontaneous intracerebral hemorrhage in central europe: A population-based study. World Neurosurg. 2016;88:306-310

\section{Tables}

Table1. Baseline data between blend sign group and control group

\begin{tabular}{|c|c|c|c|c|}
\hline Factors & Blend sign group(91) & Control group $₫ 151 \rrbracket$ & $x^{2} / z$ & P-value \\
\hline 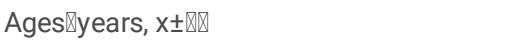 & $56.18 \pm 12.61$ & $57.58 \pm 12.77$ & -0.814 & 0.416 \\
\hline Gender $\bigotimes$ male $₫ \% \bigotimes$ & $73 \otimes 80.2 \% \bigotimes$ & 107囚70.9凶 & 2.610 & 0.106 \\
\hline History of smoking $₫ n, \% \rrbracket$ & $46 \rrbracket 50.5 \% \rrbracket$ & $75 \llbracket 49.7 \% \rrbracket$ & 0.018 & 0.500 \\
\hline History of drinking $\llbracket n, \% \rrbracket$ & $41 \varangle 45.1 \% \bigotimes$ & $67 ه 46.9 \% \rrbracket$ & 0.1072 & 0.447 \\
\hline History of hypertension $\ n, \% \bigotimes$ & $68 \otimes 74.7 \% \square$ & $110 \otimes 56.7 \% \rrbracket$ & 8.582 & 0.004 \\
\hline Anticoagulants $₫ \mathrm{n}, \% \bigotimes$ & $2 \varangle 2,2 \% \rrbracket$ & $4 \rrbracket 2.6 \% \rrbracket$ & 0.048 & 0.594 \\
\hline History of diabetes $₫ \mathrm{n} \otimes \% \bigotimes$ & $2 \llbracket 2.2 \% \rrbracket$ & $10 \otimes 6.4 \% \rrbracket$ & 2.177 & 0.119 \\
\hline Haematoma volume $\rrbracket \mathrm{ml}$, IQR】 & $37.8(33-52.5)$ & $38(31-50)$ & -0.879 & 0.379 \\
\hline Systolic pressure $\llbracket \mathrm{mmHg}, \mathrm{x} \pm \llbracket \rrbracket$ & $174.03 \pm 24.96$ & $173.33 \pm 29.53$ & -0.190 & 0.844 \\
\hline 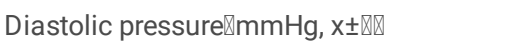 & $103.75 \pm 15.67$ & $100.63 \pm 21.70$ & 1.292 & 0.198 \\
\hline GCS on admission囚points, IQR囚 & $11 \otimes 8-13 \rrbracket$ & $11 \otimes 7-13 \bigotimes$ & -0.550 & 0.583 \\
\hline NIHSS on admission囚points, IQR】 & 16ه14-19ه & $14 \otimes 16-21 \rrbracket$ & -1.029 & 0.304 \\
\hline Time from onset to baseline CT \h, IQR】 & $4 \llbracket 2-8 \rrbracket$ & $5 \llbracket 2.5-10 \rrbracket$ & -1.860 & 0.163 \\
\hline Time from admission to surgery $\llbracket$ h, IQR $\rrbracket$ & 15区9-27》 & $15 \otimes 9.8-27 \rrbracket$ & -0.728 & 0.466 \\
\hline Duration of surgery $₫$ h, IQR $\rrbracket$ & 1.4凶1.0-1.9凶 & $1.5 \bigotimes 1.0-2.0 \rrbracket$ & -1.513 & 0.130 \\
\hline Time for removing the tube $\rrbracket$ days $₫$ IQR & $4(2-6)$ & $4 \rrbracket 3-6 \rrbracket$ & -0.121 & 0.904 \\
\hline Good outcome (n, \%) & $50 \rrbracket 54.9 \rrbracket$ & $78 \llbracket 51.7 \rrbracket$ & 0.247 & 0.619 \\
\hline Poor outcome $(n, \%)$ & $41 \rrbracket 45.1 \rrbracket$ & $73 \rrbracket 48.3 \rrbracket$ & 0.247 & 0.619 \\
\hline
\end{tabular}

GCS=Glasgow Coma Scale; NIHSS=National Institute of Health Stroke Scale

Table 2.Changes of residual haematoma volume and rate of ICH clearance during surgery

\begin{tabular}{|c|c|c|c|c|}
\hline Group & $\begin{array}{l}\text { Preoperative ICH volume } \\
\text { 『ml, IQR }\end{array}$ & $\begin{array}{l}\text { Postoperative Residual ICH } \\
\text { volume } ₫ \mathrm{ml} \text { I IQR } \mathrm{R}\end{array}$ & $\begin{array}{l}\text { Rate of ICH clearance during } \\
\text { surgery } ₫ \% \text {, IQR }\end{array}$ & $\begin{array}{l}\text { Time for removing the tube } \\
\text { \days, IQR】 }\end{array}$ \\
\hline $\begin{array}{l}\text { blend group } \\
\text { \n=91】 }\end{array}$ & $37.8(33-52.5)$ & $8 \llbracket 3.87-15 \rrbracket$ & $30.61 \rrbracket 8.67-56.67 \rrbracket$ & $4 \llbracket 2-6 \rrbracket$ \\
\hline $\begin{array}{l}\text { Control group } \\
\bigotimes n=151 \rrbracket\end{array}$ & $38.0(31-50)$ & $8 \otimes 4.5-12 \rrbracket$ & $\begin{array}{l}37.27 \rrbracket 18.98- \\
55.69 \rrbracket\end{array}$ & $4 \varangle 3-6 \rrbracket$ \\
\hline ZهP-value区 & $-0.879 ه 0.379 \rrbracket$ & $-0.456 \rrbracket 0.648 \rrbracket$ & $-0.241(0.809)$ & $-1.121 \rrbracket 0.904 \rrbracket$ \\
\hline
\end{tabular}

Table 3. Changes of GCS between the blend sign group and control group[IQR] 


\begin{tabular}{|c|c|c|c|c|}
\hline Group & On admission & One week & Two weeks & $\chi^{2} / \otimes P$-value $\rrbracket$ \\
\hline blend sign group $₫ n=91 \rrbracket$ & 11凶8-13》 & $12 \otimes 9-13 \rrbracket^{*} *$ & $13 \otimes 12-15 \rrbracket^{\&}$ & $8.627(0.013)$ \\
\hline control group $₫ n=151 \rrbracket$ & 11ه7-13区 & $12 \varangle 8-14 \rrbracket^{*}$ & $13 \rrbracket 9-15 \rrbracket^{\&}$ & $22.974(0.000)$ \\
\hline 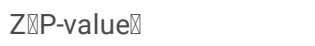 & $-1.029 \varangle 0.304 \rrbracket$ & $-0.239 \llbracket 0.811 \rrbracket$ & $-1.136 \varangle 0.256 \rrbracket$ & \\
\hline
\end{tabular}

${ }^{*}$ Compared with those on admission $\nabla \mathrm{P}<0.05 \rrbracket .{ }^{8} \mathrm{C}$ ompared with those on admission or with one week $\otimes \mathrm{P}<0.05 \rrbracket$. These results suggested that the GCS were improved one week after the surgery.

Table 4. Changes of NIHSS between the blend sign group and the control group[IQR

\begin{tabular}{|c|c|c|c|c|}
\hline Group & On admission & One week & Two weeks & F®P-value $\mathbb{Z}$ \\
\hline Blend sign group $(n=91)$ & $16 \rrbracket 14-20 \rrbracket$ & $13 \llbracket 9-17 \rrbracket^{\$}$ & $10 \rrbracket 6-13 \rrbracket^{\$}$ & $81.475(0.000)$ \\
\hline control group $\rrbracket n=151 \rrbracket$ & $16 \rrbracket 13-20 \rrbracket$ & $14 \rrbracket 10-18 \rrbracket^{\$}$ & $12 \varangle 8-15 \rrbracket^{\$}$ & $99.987(0.000)$ \\
\hline Z『P-value $\bigotimes$ & $-2.075 \bowtie 0.381 \rrbracket$ & $-1.537 \rrbracket 0.124 \rrbracket$ & $-0.654 \llbracket 0.513 \rrbracket$ & \\
\hline
\end{tabular}

\$Compared with those on admission $\otimes P<0.05 \bigotimes$. The NIHSS did not show any difference between the two groups at any time point.

Table 5 .Comparison of severe complication rate and final outcomeln,\%[

\begin{tabular}{|c|c|c|c|c|}
\hline Group & Pulmonary infection & Heart failure & Postoperative rehaemorrhage & good outcome \\
\hline Blend sign group $(n=91)$ & $19(20.9 \%)$ & $2(2.2 \%)$ & $23(25.6)^{\&}$ & $50 \rrbracket 54.9 \% \rrbracket$ \\
\hline control group $\otimes n=151 \rrbracket$ & $30(19.87 \%)$ & $7(4.6 \%)$ & $23(15.2)$ & $78(51.7 \%)$ \\
\hline$c^{2} \bowtie$ P-value & $0.036 \varangle 0.850 \rrbracket$ & $0.943 \llbracket 0.275 \rrbracket$ & 3.892(0.049) & $0.247 \rrbracket 0.358 \rrbracket$ \\
\hline
\end{tabular}

${ }^{\&}$ Compared with the control group $₫ \mathrm{P}<0.05 \rrbracket$. The rate of postoperative rehaemorrhage was increased compared with the control group. No significant differences were observed in the outcome between the two groups.

Table 6. Univariate analysis of predictors for poor outcome of patients underwent sMIS 


\begin{tabular}{|c|c|c|c|c|}
\hline Factors & $\begin{array}{l}\text { Good outcome } \\
\text { (128 patients) }\end{array}$ & Poor outcome(114 patients) & $Z / T$ & $\begin{array}{l}\text { P. } \\
\text { Vi }\end{array}$ \\
\hline Ages $₫ \mathrm{x} \pm \mathbb{\square}$ & $55.91 \pm 12.55$ & $58.43 \pm 12.81$ & 1.493 & 0 . \\
\hline $\begin{array}{l}\text { Gender『male } \mathbb{} \\
\% \rrbracket\end{array}$ & $96 \bigotimes 75.0 \% \rrbracket$ & $84 \llbracket 73.7 \% \rrbracket$ & 0.055 & 0 . \\
\hline $\begin{array}{l}\text { History of } \\
\text { smoking } \llbracket n, \% \bigotimes\end{array}$ & $63 \rrbracket 49.2 \% \rrbracket$ & $57 \rrbracket 50.4 \% \rrbracket$ & 0.0363 & 0 . \\
\hline $\begin{array}{l}\text { History of } \\
\text { drinking } \| \mathrm{n}, \% 区\end{array}$ & $51 \rrbracket 39.8 \% \rrbracket$ & $57 \rrbracket 50.0 \% \bigotimes$ & 2.517 & 0 . \\
\hline 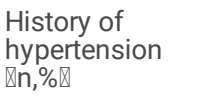 & $87 \rrbracket 68.0 \% \rrbracket$ & $91 \rrbracket 79.8 \% \rrbracket$ & 4.357 & 0 . \\
\hline $\begin{array}{l}\text { Anticoagulants } \\
\text { 『n,\%区 }\end{array}$ & $3 \llbracket 2.3 \% \rrbracket$ & $4 \llbracket 3.5 \% \rrbracket$ & 0.291 & 0 . \\
\hline $\begin{array}{l}\text { History of } \\
\text { diabetes } ₫ \mathrm{n} \otimes \% \bigotimes\end{array}$ & $4 \llbracket 3.1 \% \rrbracket$ & $9 \otimes 7.9 \% \bigotimes$ & 2.699 & 0 . \\
\hline 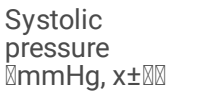 & $171.17 \pm 26.557$ & $176.32 \pm 29.113$ & 1.437 & 0 . \\
\hline $\begin{array}{l}\text { Diastolic } \\
\text { pressure } \\
\bigotimes m m H, x \pm \llbracket \square\end{array}$ & $101.07 \pm 19.733$ & $102.62 \pm 19.676$ & 0.612 & 0 . \\
\hline $\begin{array}{l}\text { GCS on } \\
\text { admission } \\
\text { 『points, IQR』 }\end{array}$ & $12(10-13.75)$ & $9(6-12))$ & -3.672 & 0 . \\
\hline $\begin{array}{l}\text { NIHSS on } \\
\text { admission } \\
\text { 『points, IQR』 }\end{array}$ & $16(13-18)$ & $17(15-22)$ & 4.105 & 0 . \\
\hline $\begin{array}{l}\text { Time from } \\
\text { onset to } \\
\text { baseline CT } \\
\text { (hour, IQR) }\end{array}$ & $5.0(2.0-9.9)$ & $4.55(2.0-9.7)$ & 0.301 & 0 . \\
\hline $\begin{array}{l}\text { ICH volume on } \\
\text { admission囚ml, } \\
\text { IQR }\end{array}$ & $36(32-50)$ & $40(30.75-52.39)$ & 0.120 & 0 . \\
\hline $\begin{array}{l}\text { Haematoma } \\
\text { ruptured into } \\
\text { ventricles } ₫ n, \% \rrbracket\end{array}$ & $43 \llbracket 33.6 \% \rrbracket$ & $47 \rrbracket 41.2 \% \rrbracket$ & 1.504 & 0 . \\
\hline $\begin{array}{l}\text { Time from } \\
\text { onset to } \\
\text { surgery } ₫ \text { h, IQR】 }\end{array}$ & 16(8.13-26.75) & $13.5(10-27)$ & 0.288 & 0 . \\
\hline $\begin{array}{l}\text { Duration of } \\
\text { surgery (h, } \\
\text { IQR) }\end{array}$ & $1.2(1.0-2.0)$ & $1.5(1.0-2.0)$ & -0.288 & 0 . \\
\hline $\begin{array}{l}\text { Blend sign } \rrbracket \mathrm{n} \rrbracket \\
\% \rrbracket\end{array}$ & $50 \rrbracket 39.1 \rrbracket$ & 41ه36.0ه & 0.247 & 0. \\
\hline $\begin{array}{l}\text { Non-blend sign } \\
\text { 『nヌ\%区 }\end{array}$ & 78 (60.9) & $73(64.0)$ & 0.247 & 0 . \\
\hline
\end{tabular}

GCS=Glasgow Coma Scale; NIHSS=National Institute of Health Stroke

Table 7. Binary logistic regression analysis of predictors for poor outcome

\begin{tabular}{|lllcll|}
\hline Variables & $\mathrm{B}$ & Wals & OR & $95 \% \mathrm{Cl}$ & $P$ \\
\hline History of hypertension & 3.170 & 2.691 & 23.800 & $0.539-1.050$ & 0.101 \\
\hline GCS on admission & 0.577 & 4.140 & 1.781 & $1.021-3.106$ & 0.042 \\
\hline NIHSS on admission & 0.522 & 4.649 & 1.686 & $1.049-2.710$ & 0.031 \\
\hline
\end{tabular}

Note: only the GCS and NIHSS on admission were associated with the poor outcome. The blend sign on initial CT has no effects on the outcome of patients who underwent a minimally invasive surgery.

\section{Figures}




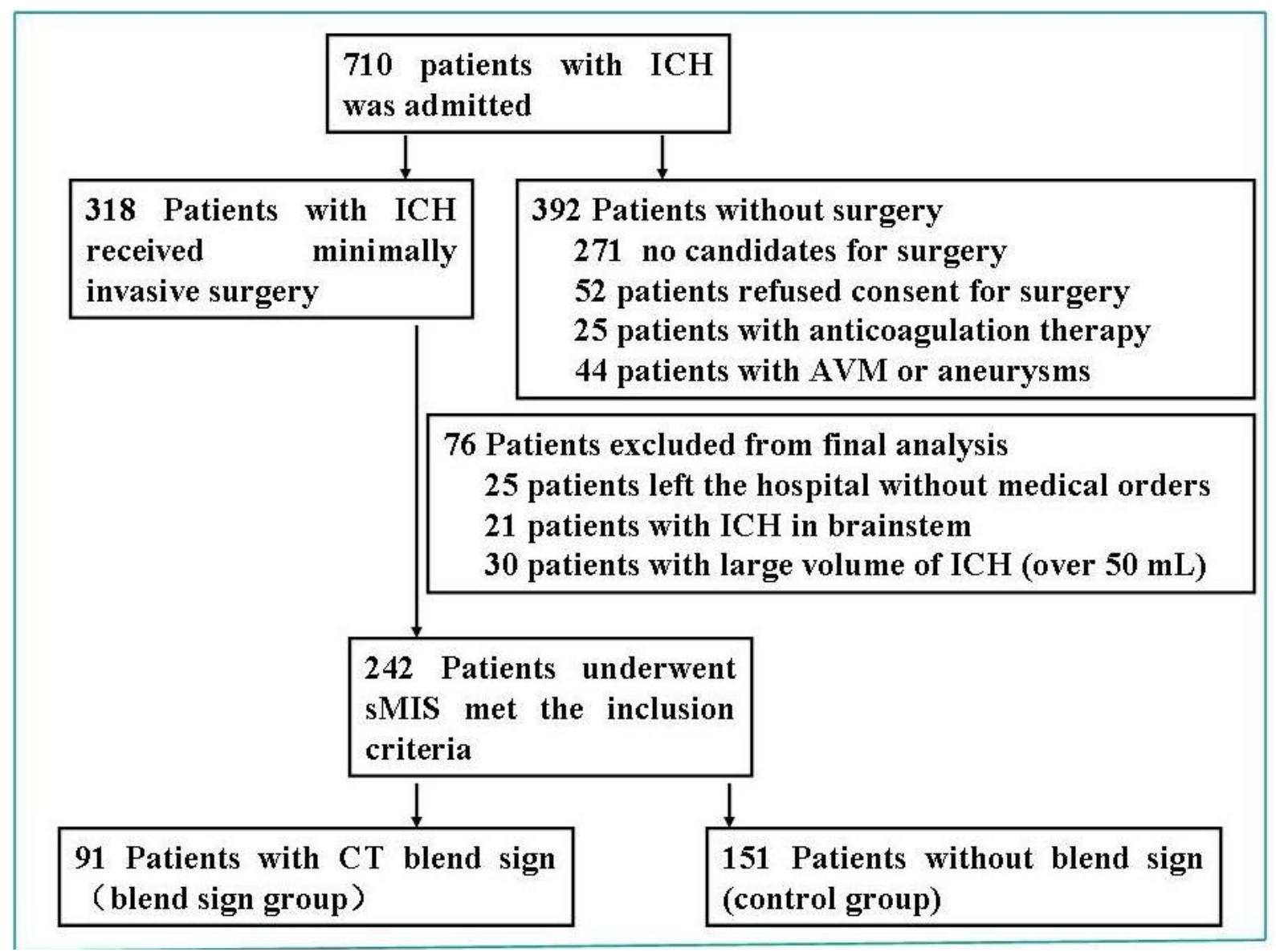

Figure 1

Flowchart of patients for selecting candidates for minimally invasive surgery A total of 710 patients with ICH were admitted. Three hundreds and eighteen patients received stereotactic minimally invasive surgery. Finally, only 242 patients with ICH met the inclusion criteria.

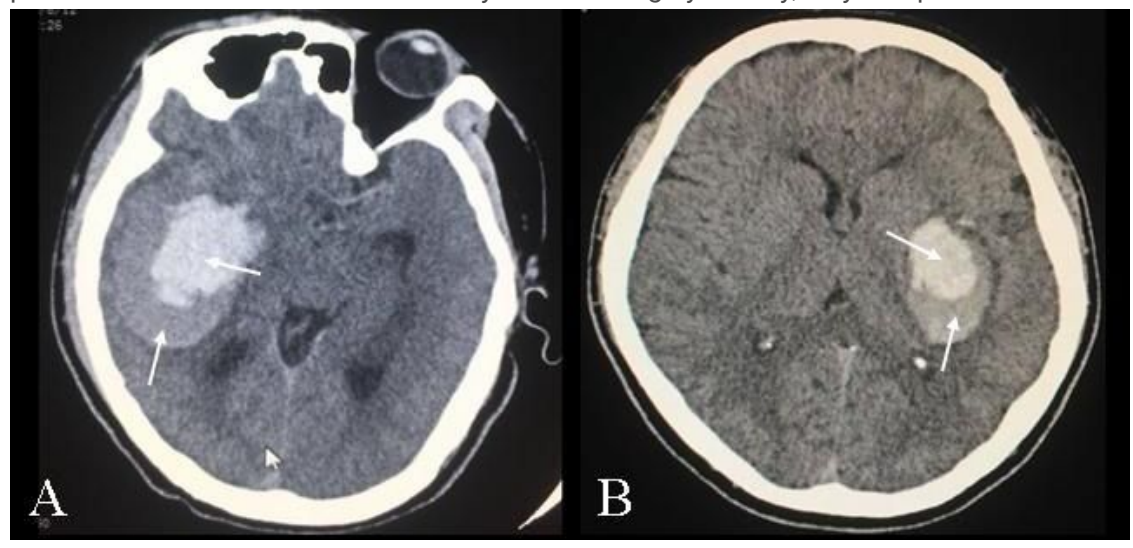

Figure 2

Blend signs on initial CT of patients with $\mathrm{ICH}$ The $\mathrm{ICH}$ was located in the right (A) or the left (B) basal ganglia. The blend signs were composed of a hyperdensity and a relative hypodensity (pointed by the arrow). The boundary of the two parts was easily identified by naked eyes. 


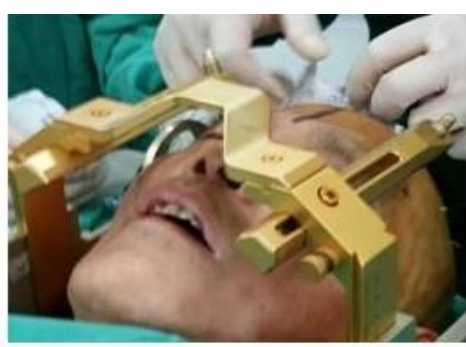

a. The headframe was fixed on the head

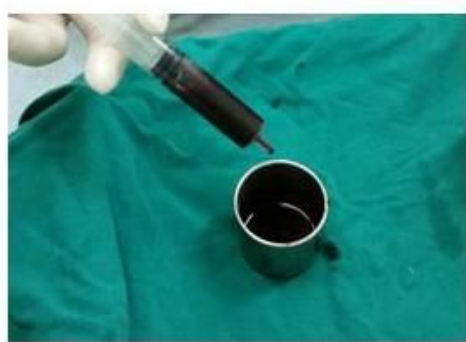

h. The aspirated liquid parts of the he ma to ma

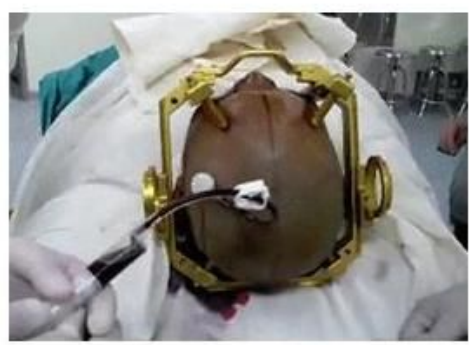

g. The intracerebral he matoma was aspirated

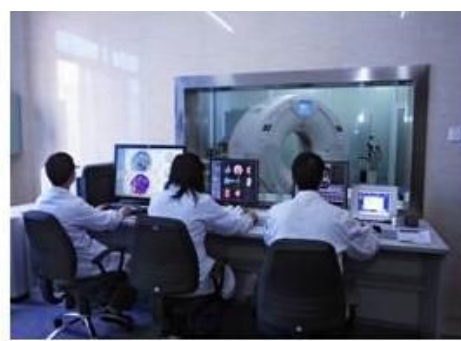

b. The patients were transferred to $\mathrm{CT}$ room

\section{Procedures of the frame- based stereotactic minimally invasive surgery}

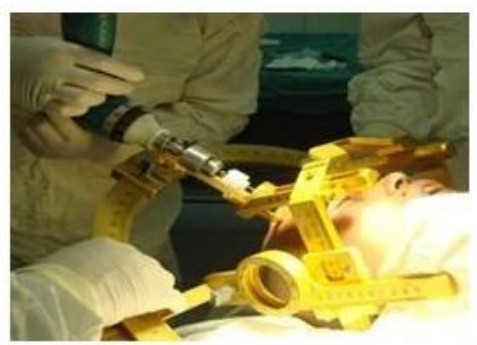

f. The puncture needle was inserted under guidance

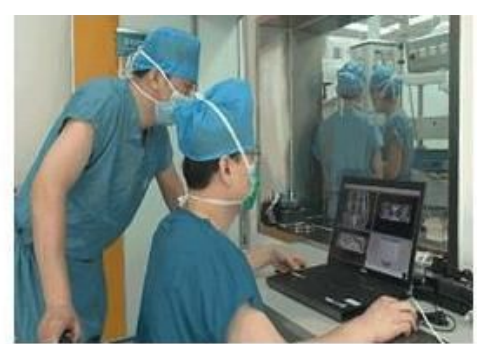

c. The coordinates were figured out by $\mathrm{CT}$ data

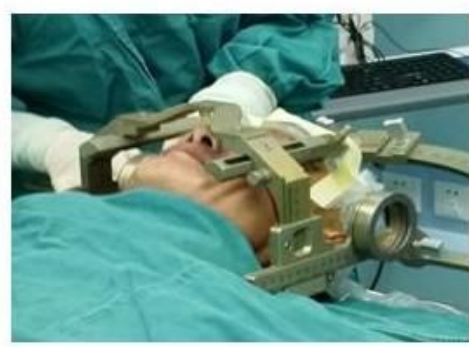

d. Surgeons installed the arc frame and guider

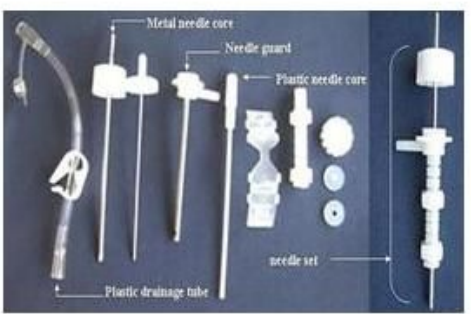

e. Transcranial p uncture needles assembly

Figure 3

Procedures for the stereotactic minimally invasive surgery A Positioning headframe was fixed on the head firstly and then the patient was transferred for CT scan to figure out the coordinates (a-c). Subsequently the arc frame and guider were fixed to the positioning headframe and a transcranial puncture needle was inserted (d-f). Finally, the liquid part of the ICH was aspirated out ( $g-h)$ 

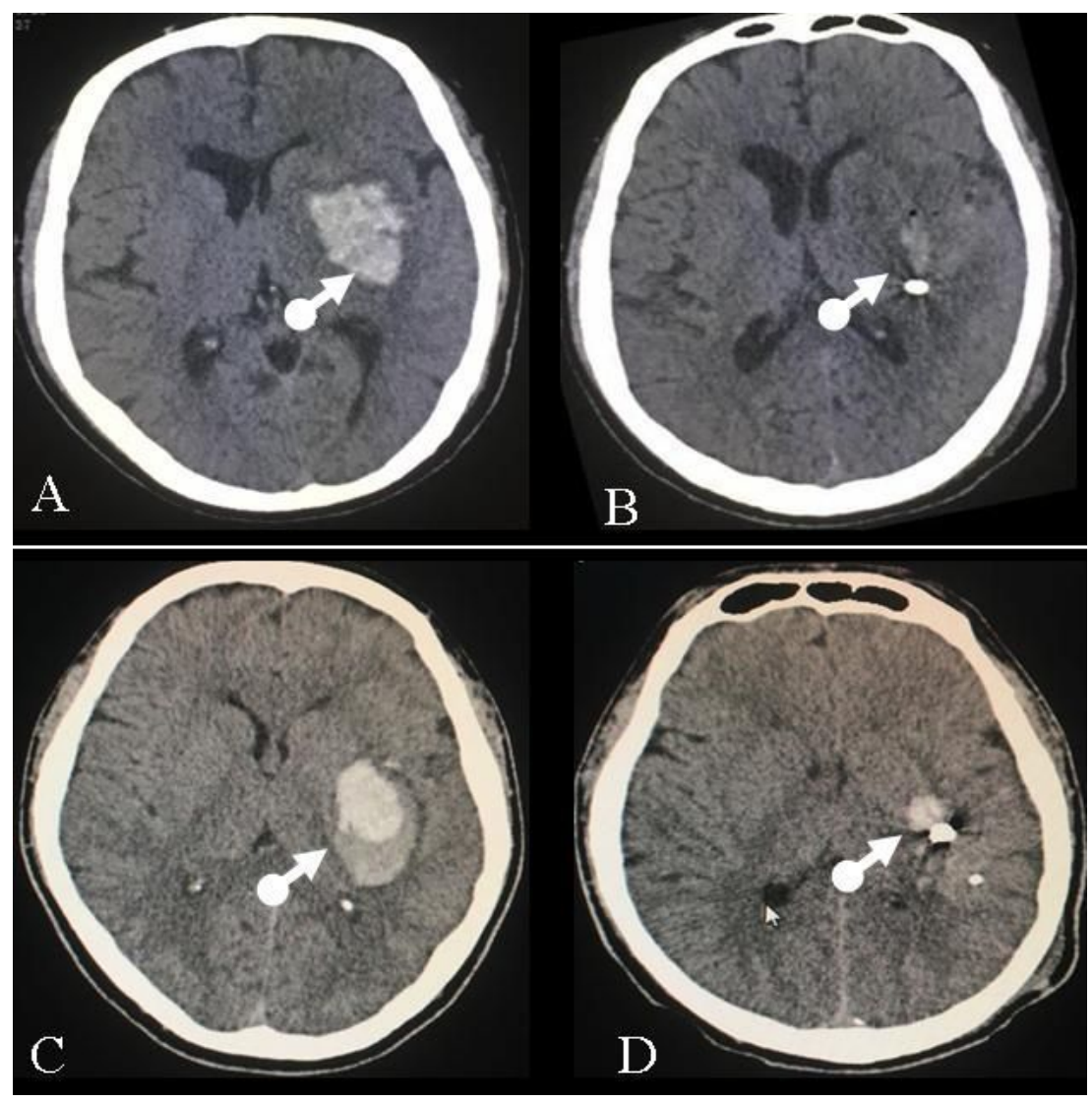

Figure 4

Changes in the haematoma volume after sMIS The haematoma volume decreased significantly after the sMIS in both the patients with non-blend signs (A-B) and the patients with blend signs(C-D). 